\title{
A gestão de informações em um haras: Uma proposta de aplicação com a tecnologia RFID
}

The management of information in a house farm: A proposal for application with RFID technology

\author{
Victor Nassar, Jonathan Ken Nishida, André Salomão \& Milton Luiz Horn Vieira
}

gestão, tecnologia da informação, RFID

\begin{abstract}
O objetivo deste artigo foi o de apresentar uma possibilidade de aplicação da tecnologia RFID para a gestão de informação em um haras, em específico para o monitoramento dos cavalos. Definiu-se o processo de controle automatizado com RFID, com a elaboração de estratégia de coleta dos dados, a seleção de equipamentos e os procedimentos a serem adotados, realizando o monitoramento de cavalos, funcionários e visitantes, bem como os locais do haras que irão receber as antenas RFID. Os dados coletados são agrupados em um aplicativo, atuando no compartilhamento das informações com os usuários. O sistema proposta agrega na identificação do animal os dados de origem, data de nascimento, tipo de alimentação e histórico de saúde, assim como o registro dos locais em que o animal circulou, auxiliando no controle de supervisão das atividades, proporcionando melhorias na segurança e na qualidade do atendimento com os clientes.
\end{abstract}

\section{management, information technology, RFID}

The objective of this paper was to present a possibility of applying RFID technology for the management of information in a horse farm, specifically for the monitoring of horses. The process of automated control with RFID was defined, with the elaboration of data collection strategy, the selection of equipment and the procedures to be adopted, the monitoring of horses, employees and visitors, as well as the locations of the farms that will Receiving the RFID antennas. The collected data is grouped in an application, acting on the sharing of the information with the users. The proposed system adds in the identification of the animal the data of origin, date of birth, type of feeding and health history, as well as the registration of the places where the animal circulated, assisting in the control of activities, providing improvements in safety and In the quality of customer service.

\section{Introdução}

O desenvolvimento tecnológico é determinante para as organizações buscarem a eficiência em suas operações, proporcionarem valor ao consumidor e, consequentemente, permanecerem competitivas no mercado. Cada vez mais, empresas utilizam tecnologias de informação para auxiliar o gerenciamento de processos, possibilitando um avanço de controle e coordenação nas suas atividades operacionais.

Em uma gestão de um haras, o administrador encontra diferentes dificuldades para efetuar um controle das informações, no que se refere ao acompanhamento e histórico da saúde dos cavalos, bem como das atividades diárias que são realizadas local. Ao mesmo tempo, há o desafio pela manutenção da qualidade no bem-estar os animais para atender os requisitos legais de fiscalização e também do público que frequenta o haras e possui cavalos no local. Com isso, ressalta-se o incentivo às aplicações de pesquisa e desenvolvimento que possam contribuir com essa gestão.

Assim, a utilização da tecnologia de comunicação sem fio RFID (Radio Frequency Identification) tem se mostrado capaz de solucionar diferentes problemas que permeiam um sistema de gerenciamento, facilitando a tomada de decisões a partir da indicação correta dos dados coletados e pela visão global das ações que estão ocorrendo (Bowersox \& Closs, 2009).

Anais do 9 CIDI e 9 CONGIC

Luciane Maria Fadel, Carla Spinillo, Anderson Horta, Cristina Portugal (orgs.)

Sociedade Brasileira de Design da Informação - SBDI

Belo Horizonte | Brasil | 2019

ISBN 978-85-212-1728-2
Proceedings of the 9th CIDI and 9th CONGIC

Luciane Maria Fadel, Carla Spinillo, Anderson Horta, Cristina Portugal (orgs.)

Sociedade Brasileira de Design da Informação - SBDI Belo Horizonte | Brazil | 2019

ISBN 978-85-212-1728-2 
Para este artigo, objetiva-se apresentar uma proposta de aplicação da tecnologia RFID para constituir um sistema de gestão em um haras, com o rastreamento individual dos cavalos, permitindo o controle de vacinas, localização espacial, tipo de alimentação e informações de origem. Da mesma forma, tem-se o monitoramento dos trajetos pelos quais cada animal passou, o tempo que ficou em cada local, as atividades que realizou, os funcionários e os visitantes que o acompanharam. A partir disso, possibilita-se a criação de um banco de dados capaz de oferecer um controle e um monitoramento preciso do histórico de cada cavalo.

\section{Contextualizando a tecnologia RFID}

A tecnologia RFID é um dos componentes que pode auxiliar na aplicação da rastreabilidade idealizada para o sistema de gestão do haras, ao participar do controle e monitoramento de dados. O RFID é uma sigla utilizada para Radio Frequency Identification. A tecnologia surgiu a partir da década de 80 e funciona como uma rede de identificação por radiofrequência, com alcance de distâncias variáveis em metros ou por proximidade total, de acordo com o chip e antena utilizado (Duroc \& Kaddour, 2012).

Para que ocorra a comunicação, é necessário que o objeto possua o chip RFID (pode ser denominado como tag RFID ou etiqueta RFID), que pode ser passivo (não possui fonte de energia) ou ativo (possui uma bateria). É por meio de uma antena que ocorre a captura de dados e o envio para o leitor instantaneamente. Um software é responsável pela conversão dos dados em informações significativas, conforme exemplifica a Figura 1.

Figura 1: Processo de funcionamento RFID.

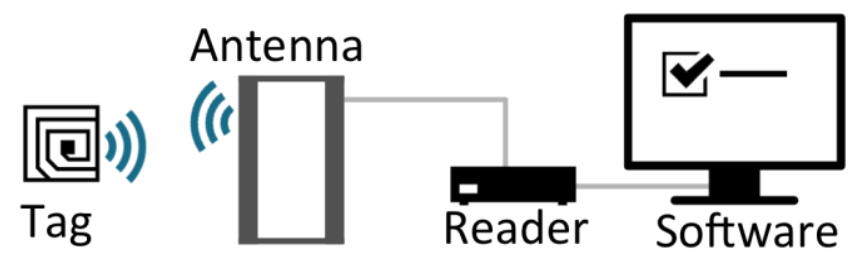

Assim, em resumo, tem-se o processo de funcionamento do sistema RFID:

1. O conjunto antena-leitor gera um campo de radiofrequência.

2. As tags que estão no campo de proximidade são ativadas.

3. A tag envia os dados de seu identificador único para a antena.

4. A antena recebe os dados da tag e envia para o software.

5. O software interpreta os dados e executa a ação programada.

O funcionamento de um sistema RFID também leva em consideração as faixas de frequência que podem ser utilizadas pela tecnologia, divididas em: baixa frequência, alta frequência e ultra alta frequência. Cada uma possui características distintas para as aplicações com RFID, conforme descreve-se a seguir:

- Baixa Frequência (Low Frequency-LF): Possui um alcance de leitura curto, o que indica a utilização para conexões de proximidade entre as etiquetas com RFID e o leitor. Assim, encontram-se aplicações voltadas para acesso de portas e catracas e controle de inventário, por exemplo. Os chips LF também possuem baixa velocidade de leitura, o que dificulta a leitura de múltiplas etiquetas simultaneamente.

- Alta Frequência (High Frequency-HF): Possui faixa de leitura entre curta e média leitura, observando-se alcance abaixo de 1 metro de distância. Os chips com frequência HF costumam ser encontrados em objetos como chaveiros e cartões, utilizados para controle de acesso, como os bilhetes de transporte público que dão 
acesso a catracas.

- Ultra Alta Frequência (Ultra High Frequency-UHF): Pode ser configurada para alcance de leitura de longas distâncias, podendo chegar a até dezenas de metros, de acordo com a antena utilizada e o tipo de tag (se ativa ou passiva). Com isso, a frequência UHF é muito utilizada no setor de transporte, para o monitoramento de veículos em estradas ou acesso a catracas em estacionamentos.

Entre as vantagens do uso da tecnologia RFID, os autores Costa et al. (2010), Freiberger \& Bezerra (2010), Navarro et al. (2008), Soares et al. (2008), Rosa (2007) destacam as seguintes características: Captura de dados de produtos à distância e que estão em movimento; Identificação sem a necessidade de contato ou visão do produto; Durabilidade das etiquetas; Alta capacidade de memória, leitura e escrita; Leitura de múltiplas etiquetas simultaneamente; Precisão na transmissão dos dados.

Desse modo, a tecnologia RFID pode ser utilizada com o objetivo de identificação ou rastreamento de objetos, aplicações do setor logístico, de supermercados, transporte ou cargas. Apenas é necessário que o produto possua a etiqueta com RFID e múltiplos dados podem ser capturados de uma vez pelo leitor, mesmo que os produtos estejam em movimento, sem a necessidade de contato ou visão do produto (Chao, Yang \& Jen, 2007; Freiberger \& Bezerra, 2010; Soares et al. 2008; Sun, 2012).

\section{Procedimentos}

Pode-se inferir por Gil (2009) que este trabalho caracteriza-se como descritivo, pois há a intenção de descrever particularidades de determinados eventos, analisando os dados com o intuito de estabelecer relações. No caso desta pesquisa, tem-se a finalidade de apresentar uma proposta de aplicação da tecnologia RFID na gestão de um haras.

A pesquisa delineada para a aplicação de uma proposta de rastreabilidade para uma gestão de informações de procedimentos em um haras. Para tanto, considerou-se a definição dos agentes que irão compor o sistema de rastreabilidade, os fluxos de informações e bases de dados, as necessidades para o controle, os meios humanos, técnicos e tecnológicos.

Em um primeiro momento, foi realizada a coleta de informações sobre a situação do sistema de gestão do haras. Buscou-se estabelecer um panorama dos processos aplicados no local, a fim de poder ter uma noção ampla de como são realizados os procedimentos internos, qual a infraestrutura existente, as necessidades de controle de dados, as tecnologias utilizadas, dificuldades e oportunidades para novas implementações.

Após, definiu-se como os equipamentos com RFID estariam inseridos no processo, seja com leitores fixos ou móveis, pulseiras, cartões de identificação ou etiquetas, que deveriam estar vinculados ao ambiente, aos cavalos, objetos, visitantes e funcionários participantes em cada momento.

Em seguida, foi desenvolvido o processo de controle automatizado com RFID, com a elaboração de estratégia de coleta dos dados, a seleção de equipamentos que melhor se adequam a cada momento e os procedimentos a serem adotados, a fim de garantir a eficiência no monitoramento e precisão no controle de informações do haras. Por fim, estabeleceu-se os dados que necessitariam fazer parte do sistema de gestão do aplicativo, indicando as funcionalidades pertinentes e que fazem relação com a coleta obtida por meio da tecnologia RFID aplicada ao processo.

\section{Aplicação}

A aplicação da proposta é dividida em duas etapas, com a definição dos processos do RFID no haras e o modo como as funcionalidades são destacadas no software. 


\section{Definição dos processos com RFID no Haras}

Para cada um dos elementos a serem monitorados foram analisadas as necessidades correspondentes, a fim de poder definir o tipo de tag RFID que melhor poderia ser utilizada. Para tanto, foi considerado o fluxo de movimentação no haras, para que se pudesse estabelecer posteriormente os processos de monitoramento. Após a definição dos processos de monitoramento, com as respectivas necessidades, foram determinadas as tags RFID UHF utilizadas, bem como a antena responsável pela leitura dos dados. Foi utilizada a frequência UHF, pois se busca adotar o alcance de leitura regulável a longas distâncias.

Os processos com RFID dividem-se em: 1) Monitoramento de Cavalos; 2) Monitoramento de Funcionários; 3) Monitoramento de Visitantes; 4) Monitoramento de Locais.

\section{Monitoramento dos cavalos}

Ao iniciar no haras, cada cavalo recebe uma identificação própria, por meio de um chip RFID, que agrupará os dados do animal, como procedência, raça e dono, além de servir para o controle de vacinação e demais procedimentos no haras. Com a integração da chip RFID do cavalo e as Antenas RFID nos locais, pode-se ter um monitoramento sobre as áreas que o animal circulou.

O chip definido foi o modelo UHF adesivo (Figura 2), no tamanho de $22 \times 75 \mathrm{~mm}$, que pode ser incluído na rédea ou touca do cavalo, para que fique posicionado próximo à cabeça, o que facilita a leitura das antenas, além de não gerar incômodo ao animal.

Com isso, espera-se oferecer um processo que proporcione aos donos dos cavalos o acompanhamento em tempo real do local em que o cavalo se encontra e atividades que realizou, como alimentação, vacina, banho e passeio, gerando um conhecimento do histórico de cada animal.

\section{Monitoramento dos funcionários}

Os funcionários também podem ser monitorados no haras. De acordo com o cargo, determinados funcionários podem possuir identificação com cartões com RFID (Figura 2). Cada cartão agrupará histórico sobre os locais em que a pessoa circulou, com dias e horários, facilitando no acompanhamento da jornada de trabalho e atividades que realizou no haras.

\section{Monitoramento dos visitantes}

Os visitantes também podem ser monitorados no haras, a partir do cadastro na entrada e utilização de cartão de RFID para acesso (Figura 2). Com isso, tem-se registro dos locais em que o visitante se encontra e cavalos a que estão vinculados. Com o cartão, pode-se ainda restringir 0 acesso a áreas proibidas.

Figura 2: Tipos de Tags e Antena RFID para o Monitoramento das pessoas

Tag Cavalo

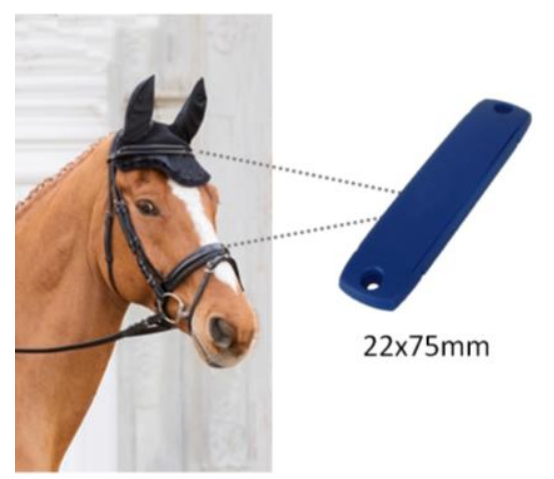

Tag Pessoas

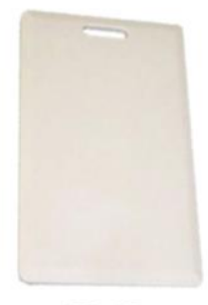

$55 \times 85 \mathrm{~mm}$
Antena RFID

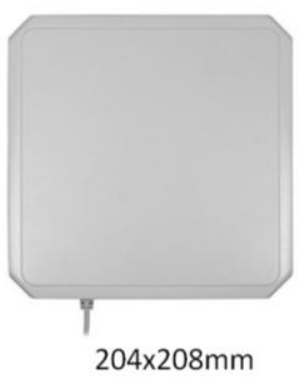




\section{Monitoramento dos locais}

Cada ambiente do haras é equipado com antenas de RFID (Figura 2), a fim de monitorar a movimentação dos cavalos, visitantes e funcionários. Assim, pode-se criar um monitoramento em tempo real da movimentação de cada área do haras, como o depósito de equipamentos, o lavador, o curral, as baias dos cavalos, o redondel, a selaria, os piquetes, etc. (Figura 3). Com isso tem-se um histórico de entrada e saída dos locais, a fim de auxiliar o acompanhamento das atividades de cada um dos cavalos monitorados, propiciando, com isso, uma maior segurança e um maior controle das informações sobre o que acontece na rotina do haras.

Figura 3: Monitoramento RFID dos locais no Haras

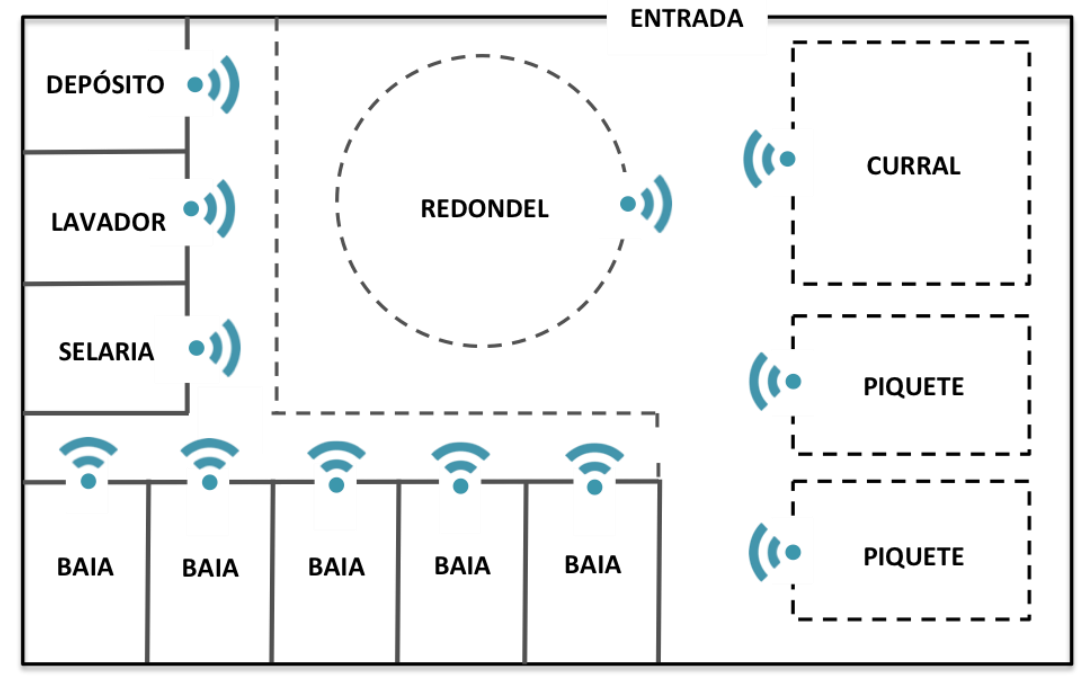

\section{Aplicativo}

Para a execução do processo, além dos elementos RFID envolvidos, há o aplicativo para realizar a gestão dos dados coletados. Com isso, pode-se oferecer uma rastreabilidade no processo que seja capaz de auxiliar na tomada de decisões, em intervenções localizadas e no conhecimento preciso da movimentação em cada local e do histórico de cada cavalo (Figura 4).

Dessa forma, o sistema divide-se em: 1) Monitoramento dos Cavalos; 2) Monitoramento de Locais com antena RFID.

\section{Monitoramento dos cavalos}

Os dados do aplicativo são abastecidos com os monitoramentos RFID, que enviam para o sistema de forma automatizada, além do próprio cadastro manual por parte dos funcionários. Assim, tem-se um sistema de gestão responsável pelo histórico do cavalo no haras. As categorias de informações, com os respectivos dados armazenados, são divididas em:

- Dados do animal: Foto, Nome, Raça, Idade, Origem, Categoria, Criador, Proprietário, Características físicas, Código Identificador RFID.

- Alimentação: Tipo de Ração, Quantidade utilizadas, Monitoramento RFID dos períodos de alimentação.

- Saúde: Calendário de vacinação do animal, Registro do estado de saúde, Remédios e Tratamentos utilizados, Monitoramento RFID dos períodos em que ocorreu a vacinação.

- Ciclo Reprodutivo: Acompanhamento da Égua no período de reprodução, com dados de Data de Inseminação, Identificação do Sêmen utilizado, Vacinações específicas, Consultas Veterinárias. 
- Atividades: Registro das atividades realizadas e Monitoramento RFID dos locais em que o cavalo passou (Baias, Selaria, Passeios, Lavador, Piquetes).

Figura 4: Dados de monitoramento do cavalo

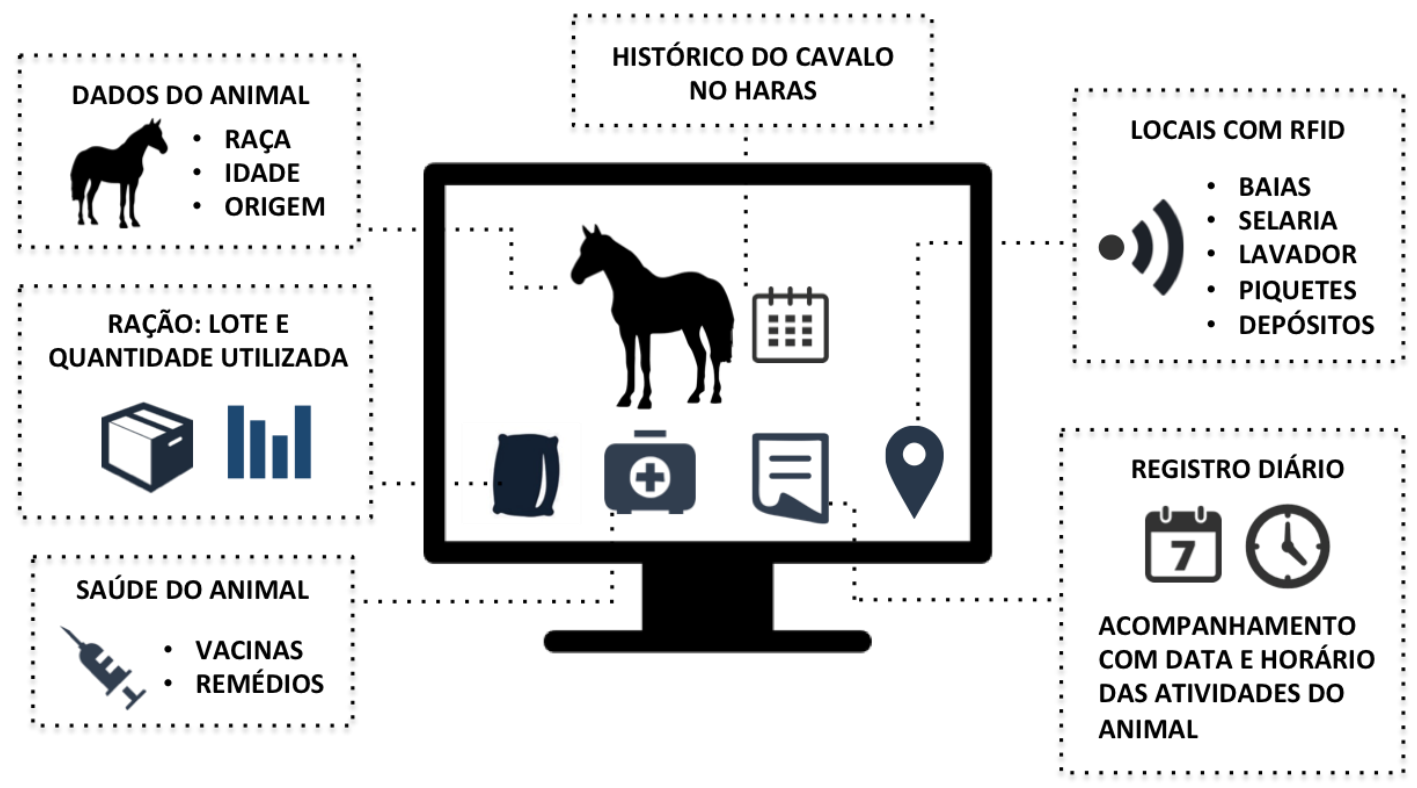

\section{Monitoramento dos locais com antenas RFID}

Outra seção do aplicativo é a de monitoramento dos locais que possuem antenas RFID. Em cada ambiente monitorado, há a leitura RFID no momento em que alguma pessoa ou cavalo transita no raio do sinal. Com isso, tem-se a categoria de informação:

- Registro: Para o acompanhamento em tempo real da movimentação de pessoas e cavalos nos locais que possuem antena RFID instaladas, como baias, depósitos, selarias, entrada do haras. Além disso, informa datas e horários em que os animais, visitantes e funcionários circularam pelas passagens dos locais.

\section{Considerações finais}

O objetivo deste artigo foi o de apresentar uma possibilidade de aplicação da tecnologia RFID para a gestão de informação em um haras, em específico para o monitoramento dos cavalos. Os dados coletados são agrupados em um aplicativo, atuando no compartilhamento das informações com os usuários.

A rastreabilidade com chips RFID em um haras é aplicada ao efetuar a identificação eletrônica do cavalo, agregando a dados como a origem do animal, data de nascimento, tipo de alimentação, histórico de saúde e outras informações pertinentes. Com a instalação de antenas RFID em diferentes locais de passagem, pode-se ter um histórico preciso da presença dos animais em cada área, auxiliando no controle de supervisão das atividades.

Além disso, com o monitoramento em locais do haras associado à identificação dos cavalos, funcionários e visitantes, tem-se a emissão de alertas e/ou proibição de acesso em casos de movimentação em áreas restritas. Dessa forma, pode-se auxiliar a segurança dos internos em locais com controles rígidos, a partir do estabelecimento de diferentes níveis de permissão de acesso.

Dessa forma, pode-se atuar proporcionando melhorias na segurança dos animais e na qualidade do atendimento com os clientes, além de agilidade e eficiência na gestão do haras. A rastreabilidade com RFID cria uma base de conhecimento integrado ao ciclo de vida dos animais. Com isso, tem-se não apenas a contribuição para o controle de informações do haras, 
mas para o estabelecimento de certificações e padronização de normas, com a criação de novos protocolos que visem a implementação de boas práticas agropecuárias.

\section{Agradecimento}

Agradecemos o apoio e financiamento de Capes, CNPq e Universidade Federal de Santa Catarina, bem como à equipe do DesignLab/UFSC pela colaboração com as pesquisas.

\section{Referências}

Bowersox, D. J., \& Closs, D. J. (2009). Logística empresarial: o processo de integração da cadeia de suprimentos. São Paulo: Atlas.

Chao, C., Yang, J., \& Jen, W. (2007). Determining Technology Trends and Forecasts of RFID by a Historical Review and Bibliometric Analysis from 1991 to 2005. Technovation. Taiwan, 27, pp.268-279.

Costa, F.; Silva, H.; Cavalcante, J. \& Araujo, A. (2010). Modelo De Simulação Computacional para Avaliar a Implantação da Tecnologia de Identificação Por Rádio Frequência (RFID) em uma Indústria de Telefones Celulares. Anais do XXX Encontro Nacional de Engenharia de Produção (ENEGEP), São Carlos, Outubro;

Duroc, Y.,\& Kaddour, D. (2012). RFID Potential Impacts and Future Evolution for Green Projects. Energy Procedia, Elsevier/Science Direct, 18, pp.91-98.

Freiberger, A., \& Bezerra, M. B. P. (2010). RFID e Seus Impactos na Logística. Março.

Gil, A. C. (2008). Métodos e técnicas de pesquisa social. 6 ed São Paulo: Atlas.

Navarro, C.; Grillo, A., \& Lima, R. (2008). Análise e Proposição de Melhorias no Processo Logístico em uma Multinacional de Tecnologia de Informação e Automação. Anais do XXVIII Encontro Nacional de Engenharia de Produção. Rio de Janeiro-RJ: Outubro;

Rosa, L. A. (2006). Aplicação do RFID na Cadeia Logística. 63f. Monografia (MBA em Tecnologia da Informação). Universidade de São Paulo (USP). Escola Politécnica. São Paulo-SP.

Soares, R. S.; Comucci, T. L.; Dos Santos, T. C. Almeida, T. B., \& Leite, P. R. (2008). O Impacto da Tecnologia de Etiqueta Inteligente (RFID) na Performance de Cadeias de Suprimentos - Um Estudo no Brasil. Revista Jovens Pesquisadores, ano V, n. 9, jul./dez.;

Tajima, M. (2007). Strategic Value of RFID in Supply Chain Management. Journal of Purchasing \& Supply Management 13, p.261-273.

\section{Sobre o(a/s) autor(a/es)}

Victor Nassar, Dr., UFSC, Brasil <victornassar@gmail.com>

Jonathan Ken Nishida, M. S., UFSC, Brasil <jounishida@gmail.com>

André Salomão, Mestrando, UFSC, Brasil <andresalomao3d@gmail.com>

Milton Luiz Horn Vieira, Dr., UFSC, Brasil <milton.vieira@ufsc.com> 